\title{
Hormone Receptor DNA-Binding Domain
}

National Cancer Institute

\section{Source}

National Cancer Institute. Hormone Receptor DNA-Binding Domain. NCI Thesaurus. Code C13428.

One of three structural motifs observed in DNA binding domains of zinc proteins.

Originally identified in the glucocorticoid receptor, it's also known as the C4 zinc finger, or the zinc twist. Each of two zinc atoms is bound to four cysteine residues; the zinc twist is represented by a helical DNA recognition site located between the two zinc atoms. 